\title{
Case Report: Case of Coronary Stent Longitudinal Deformation
}

\author{
Suresh Yerra ${ }^{1} \quad$ Rama Kishore $Y^{1}$ \\ ${ }^{1}$ Department of Cardiology, Nizam's Institute of Medical Sciences, \\ Panjagutta, Hyderabad, India
}

Address for correspondence Suresh Yerra, DM (Cardiology), Department of Cardiology, Nizam's Institute of Medical Sciences, Panjagutta, Hyderabad, 500082, India (e-mail: Yerrasuresh3@gmail.com).

Ind J Car Dis Wom 2019;4:146-149

\author{
Abstract \\ Keywords \\ - longitudinal stent \\ deformation (LSD) \\ - drug-eluting stents \\ (DES) \\ - quantitative coronary \\ analysis (QCA) \\ - bioabsorbable poly- \\ meric scaffolds (BRS)
}

The foreshortening of a stent also known as longitudinal stent deformation (LSD) is a potential complication of present generation drug-eluting stents (DES) that had thin struts design, and it was most commonly reported in the everolimus-eluting Promus Element stent. However, there are limited reports of migration and dislodgement of a DES shortly after its deployment. So, we describe a case of a DES foreshortening 1 and half months after its deployment. While performing complex interventions, careful handling and gentle manipulation of procedure-related devices and use of imaging modalities such as optical coherence tomography, intravascular ultrasound, and adjunctive balloons for complete lesion cover and optimal stent apposition may significantly reduce the occurrence of LSD and related complications.

\section{Introduction}

The foreshortening of a stent also known as longitudinal stent deformation (LSD) is a potential complication of present generation drug-eluting stents (DES) that had thin struts design, and it was most commonly reported in the everolimus-eluting Promus Element stent. It has also been reported intermittently in other types of stent platforms, thereby suggesting that there might be risk factors other than the stent design for LSD. ${ }^{1-3}$ However, there is limited existing data on the most predominant predictors of LSD, which was defined by quantitative methods such as the quantitative coronary analysis (QCA) and unclear long-term clinical implication of LSD. Stent migration and embolization are a rare complication of coronary intervention and might create a stressful situation for the operators/interventional cardiologists. ${ }^{4-6}$ In post cardiopulmonary resuscitation ${ }^{7}$ cases, there are case reports that showed complications such as distortion, compression, dislodgement, and migration of large endovascular stents. Multiple trials have showed that increased rate of stent deformation result in stent thrombosis with bioabsorbable polymeric scaffolds (BRS) (.9 $^{8}$ and it may be explained by malapposition from weak polymer materials in BRS and result in asymmetric material degradation from microstructural damage due to local stress concentration from the stent crimping, which is less often reported in stents with nonabsorbable polymeric scaffolds. However, there are limited reports of migration and dislodgement of a DES shortly after its deployment. So, we describe a case of a DES foreshortening (LSD) 1 and half months after its deployment.

\section{Case Details}

A 44-year-old male patient reformed smoker and occasional alcoholic with no comorbidities presented to a private hospital at Khammam with acute inferior wall ST-elevation myocardial infarction. He underwent coronary angiogram that showed double vessel disease (left anterior descending|right coronary artery $[\mathrm{LAD} / \mathrm{RCA}]$ ), following which he underwent primary percutaneous coronary intervention $(\mathrm{PCI})$ to RCA on 21 May 2019 (-Figs. 1 and 2). In June 2019, patient was referred to our institute for the residual lesion (LAD) revascularization. The patient was asymptomatic at the time of presentation. He underwent a coronary angiogram, which showed complete occlusion of the RCA stent and stenosis proximal to the RCA stent. On comparing with the previous $\mathrm{PCI}$ angiograms with the present coronary angiography views and QCA, it was suggestive of the stent foreshortening (LSD) and doubtful short distance migration (-Figs. 3 and $\mathbf{4}$ ); as
DOI https://doi.org/ 10.1055/s-0039-3399617
C2019 Women in Cardiology and Related Sciences
License terms

()(1) $\Theta \circledast$ 


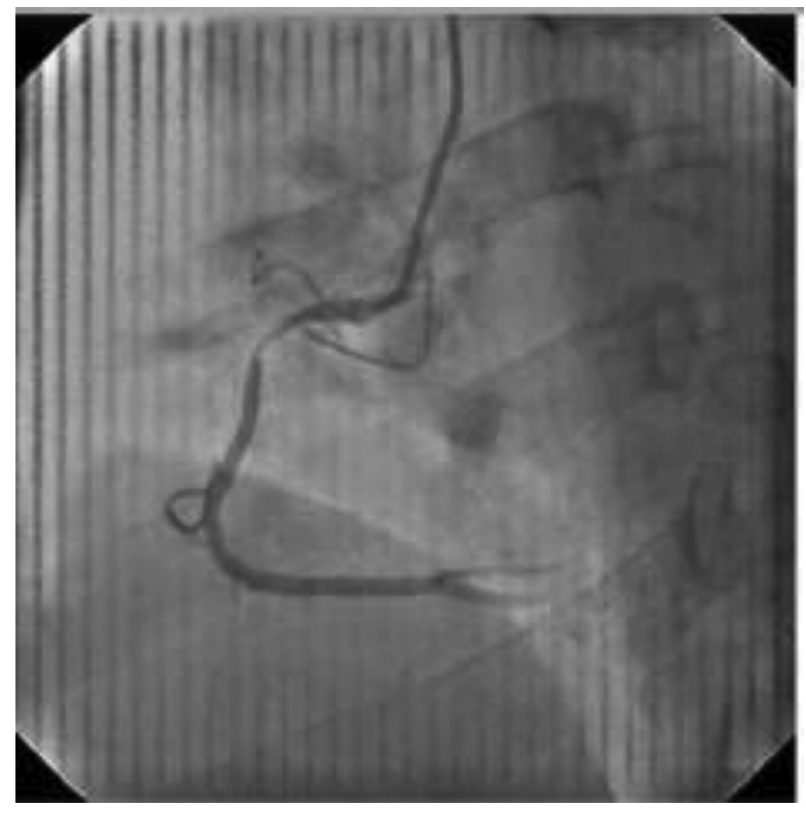

Fig. 1 Angiographic view of right coronary artery at the time of initial presentation with inferior wall myocardial infarction.

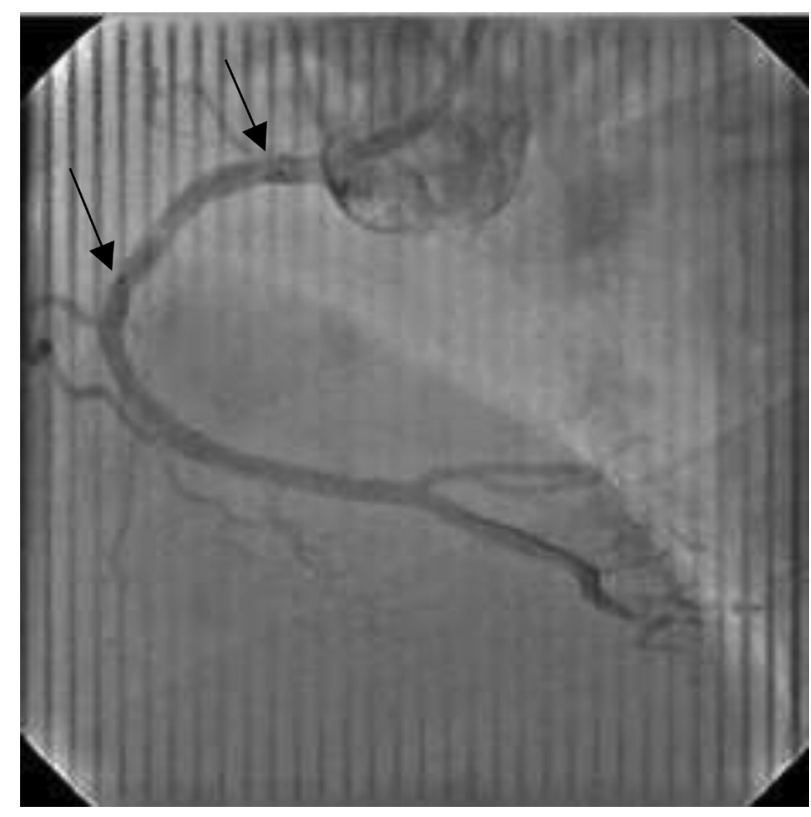

Fig. 2 Positioning of a stent with the help of balloon markers (arrows) and final angiographic view after stent deployment.

the details of the previously deployed stent were not available with the patient, we could not calculate the QCA of the initially deployed stent. We can notice that both longitudinal and horizontal deformation had occurred along with a mild degree of stent migration. The patient underwent PCI to LAD and subsequently planned PCI to RCA. With the help of fine cross coronary microguide catheter and Asahi Gaia 2 wire proximal RCA lesion was crossed and predilated sequentially with $10 \times 10 \mathrm{~mm}, 1.5 \times 10 \mathrm{~mm}$ Tazuna balloons and $30 \times 17 \mathrm{~mm}$ Apollo NC balloon and stented with Xience Xpedition $3.0 \times 48 \mathrm{~mm}$ DES (-Fig. 5).

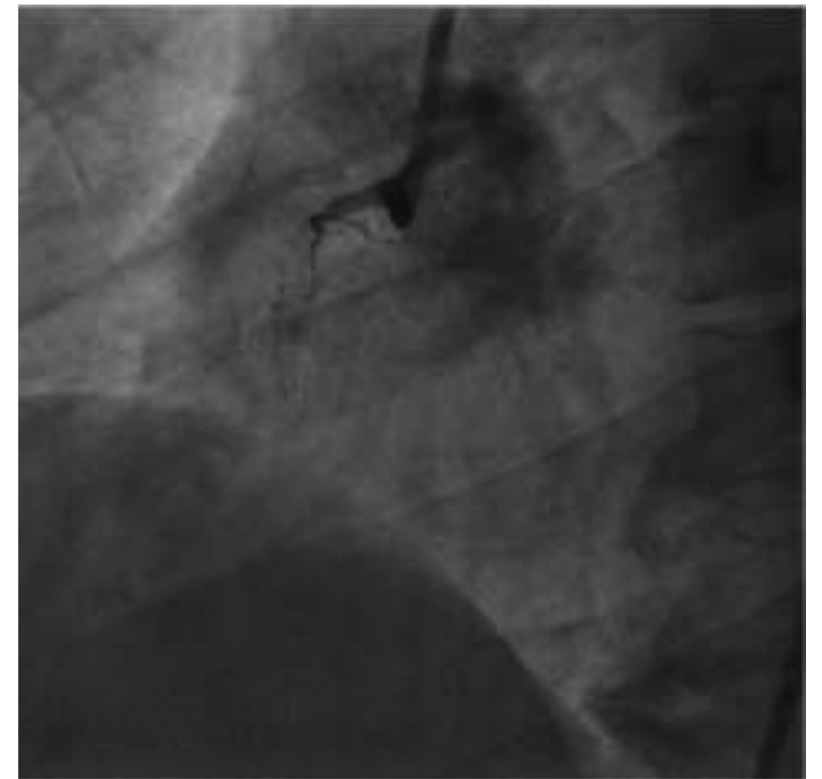

Fig. 3 Coronary angiogram done 1 and a half month after right coronary artery stent deployment.

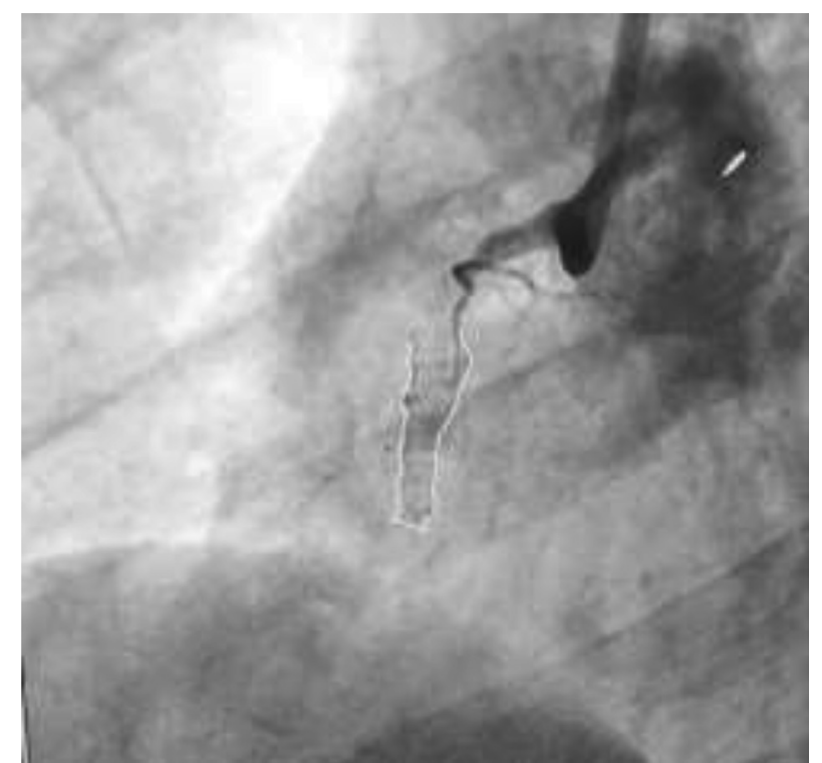

Fig. 4 Quantitative coronary analysis. Length: $12.51 \mathrm{~mm}$; minimum diameter: 1.82; maximum diameter: 4.30; mean diameter: 2.85 .

\section{Discussion}

The multiple studies have shown that ballooning or stenting of the provisional side branch, downstream complex intervention, and use of imaging devices such as optical coherence tomography (OCT) and intravascular ultrasound (IVUS) were the predictors and shown to be independently associated with LSD based on QCA. Arnous et al ${ }^{10}$ have shown that multivessel stenting, the use of GuideLiner catheter, and postdilation ballooning were the predictors of LSD. Use of extra support guidewire or guiding catheter for complex 


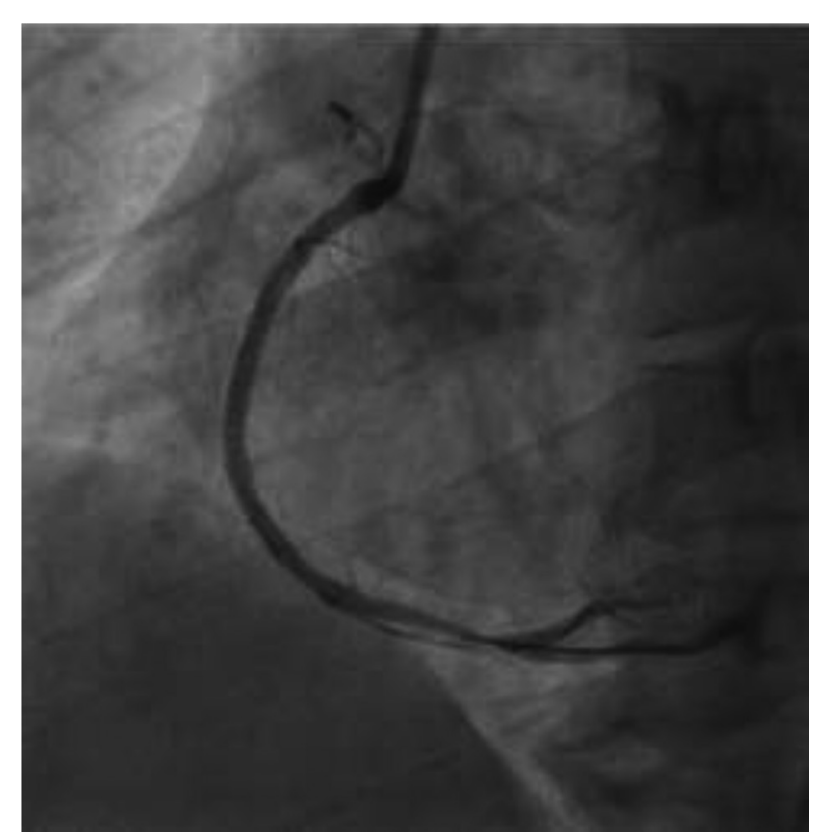

Fig. 5 Final angiographic views after restenting the proximal right coronary artery.

PCIs or passage of a second stent had been significantly associated with LSD in another study. ${ }^{1}$ In addition, aggressive manipulation of guiding catheter, use of imaging-guided PCI under OCT or IVUS, and serial dilation with multiple balloons have been suggested as major predictors associated with the occurrence of LSD. ${ }^{11,12}$

In cases of nonapposition of the proximal portion of the stent to the vessel wall, use of secondary devices in such cases may increase the chance of contact with unopposed struts especially when wire bias exists resulting in the occurrence of LSD. ${ }^{10}$ Studies have shown that careful handling and gentle manipulation of procedure-related devices such as guiding catheters, backup supporting catheters, OCT, IVUS, and adjunctive balloons may significantly reduce the occurrence of LSD. Stents deployed in LMCA and Osteo-Proximal LAD are more prone to LSD by longitudinal forces, because of frequent malapposition of stents due to the under-sizing of stents, and because of the frequent contact with the guiding catheter during insertion and pullback of various supporting devices, it is especially common when there is poor alignment of the stent with the coronary artery. ${ }^{10,13}$ Theoretically, LSD could result in malapposition of the stent and incomplete coverage of plaque-causing early stent thrombosis and in-stent restenosis, which may account for the higher incidence of complications and adverse clinical outcomes.

Detection of subtle LSD by visual estimation of angiography is difficult, and it may result in incomplete lesion coverage and deformation of malapposed stent struts when angiographically nonsignificant LSD occurs. Therefore, confirmation of optimal stent apposition and complete lesion cover by the stent may minimize the future adverse events when LSD is suspected. Coronary stent migration and dislodgment are a critical complication of PCI rarely occurring with incidence varying between 0.31 and $8.2 \%$. Dislodgement of the stent from the stent-balloon assembly unit can occur during the pullback, into the guide catheter before stent deployment. Complex coronary interventions and extremely angulated, highly calcified plaques with inadequate predilation or interference from previously deployed stents are more prone to this complication. There are reports of coronary artery stent displacement during PCI with the use of intracoronary vasodilator drugs such as nitroglycerine. ${ }^{14}$ Incidence of LSD has been decreasing with the development of advanced hardware and improved PCI techniques; however, we continued to see reports of LSD, stent migration, and embolization with the use of premounted DES during PCI. ${ }^{4,15,16}$ In this case, we had noticed that both longitudinal and horizontal deformation of the previously deployed stent has occurred along with mild stent migration. Horizontal deformation of the implanted stent is known to occur at the native tightest portion of the stent, which had been demonstrated in this case also. Because of this, the stent was not covered in the native tightest site, leading to total occlusion at that site. This is the first case in our experience regarding LSD, horizontal deformation, and short-distance migration of a DES within 1 and half months after its deployment.

\section{Limitations}

Details of the previously deployed stent details are not known to know its exact size, and we could not calculate the QCA that is a predominant limitation of this case report, and due to the financial limitations of the patient, we could not use intracoronary imaging devices such as OCT/IVUS to know the exact cause of stent deformation that is another predominant limitation.

\section{Conclusion}

LSD, horizontal deformation, dislodgement, and migration of coronary stents are infrequent, but critical complications of coronary interventions. Complex coronary interventions with highly calcified lesions and extremely angulated vessels are more prone to such complications. Hence, while performing such interventions careful handling and gentle manipulation of procedure-related devices and use of imaging modalities such as OCT, IVUS, and adjunctive balloons for complete lesion cover and optimal stent apposition may significantly reduce the occurrence of LSD and related complications. As the horizontal deformation of the stent is known to occur at the native tightest portion of the stent that has been demonstrated in this case, careful pre- and postdilation is required with appropriate size, noncompliant balloons to achieve optimal results thereby minimizing such complications.

\section{Conflict of Interest}

None declared. 


\section{References}

1 Guler A, Guler Y, Acar E, et al. Clinical, angiographic and procedural characteristics of longitudinal stent deformation. Int J Cardiovasc Imaging 2016;32(8):1163-1170

2 Hanratty CG, Walsh SJ. Longitudinal compression: a "new" complication with modern coronary stent platforms-time to think beyond deliverability? EuroIntervention 2011;7(7):872-877

3 Mamas MA, Williams PD. Longitudinal stent deformation: insights on mechanisms, treatments and outcomes from the Food and Drug Administration Manufacturer and User Facility Device Experience database. EuroIntervention 2012;8(2):196-204

4 Colkesen AY, Baltali M, Acil T, et al. Coronary and systemic stent embolization during percutaneous coronary interventions: a single center experience. Int Heart J 2007;48(2):129-136

5 Aydin M, Sayin MR. Successful coronary stent retrieval from the saphenous vein graft to right coronary artery. Case Rep Med 2009;2009:718685

6 Nikolsky E, Gruberg L, Pechersky S, et al. Stent deployment failure: reasons, implications, and short- and long-term outcomes. Catheter Cardiovasc Interv 2003;59(3):324-328

7 Haas NA, Happel CM, Jategaonkar S, et al. Compression, distortion and dislodgement of large caliber stents in congenital heart defects caused by cardiopulmonary resuscitation: a case series and review of the literature. Clin Res Cardiol 2014;103(9):719-725

8 Moriyama N, Shishido K, Tanaka Y, et al. Neoatherosclerosis 5 years after bioresorbable vascular scaffold implantation. J Am Coll Cardiol 2018;71(17):1882-1893
9 Jinnouchi H, Torii S, Sakamoto A, Kolodgie FD, Virmani R, Finn AV. Fully bioresorbable vascular scaffolds: lessons learned and future directions. Nat Rev Cardiol 2019;16(5):286-304

10 Arnous S, Shakhshir N, Wiper A, et al. Incidence and mechanisms of longitudinal stent deformation associated with Biomatrix, Resolute, Element, and Xience stents: angiographic and case-by-case review of 1,800 PCIs. Catheter Cardiovasc Interv 2015;86(6):1002-1011

11 Inaba S, Mintz GS, Yun KH, et al. Mechanical complications of everolimus-eluting stents associated with adverse events: an intravascular ultrasound study. EuroIntervention 2014;9(11):1301-1308

12 Seth A, Dhall A. Longitudinal stent shortening: the long and short of it!! Catheter Cardiovasc Interv 2013;81(5):818-819

13 Williams PD, Mamas MA, Morgan KP, et al. Longitudinal stent deformation: a retrospective analysis of frequency and mechanisms. EuroIntervention 2012;8(2):267-274

14 Celik M, Cagdas Yuksel U, Gokoglan Y. Migration of fully deployed stent after intracoronary glyceryl trinitrate administration: an unusual percutaneous coronary intervention complication. Pak J Med Sci 2013;29(3):863-865

15 Brilakis ES, Best PJ, Elesber AA, et al. Incidence, retrieval methods, and outcomes of stent loss during percutaneous coronary intervention: a large single-center experience. Catheter Cardiovasc Interv 2005;66(3):333-340

16 Eggebrecht $\mathrm{H}$, Haude $\mathrm{M}$, von Birgelen $\mathrm{C}$, et al. Nonsurgical retrieval of embolized coronary stents. Catheter Cardiovasc Interv 2000;51(4):432-440 